\title{
Reliable SPIN in Wireless Sensor Network: A Review
}

\author{
Jitender Grover ${ }^{1}$, Mohit Sharma ${ }^{2}$, Shikha ${ }^{3}$ \\ 1, 2, 3 Department of Computer Science \& Engineering, M. M. University, Sadopur, Ambala, India
}

\begin{abstract}
Wireless sensor network (WSN) is becoming famous because of its use in various applications of different areas such as environment, defense, medical, house, media and education. WSN is a structure free network where requirement of application decides the network structure, topology and routing protocols. Data transmission in sensor network is possible with the help of routing protocols. There are multiple routing protocols in WSN which provide efficient data delivery but reliability is still a major constraint of WSN. When data is transmitted from source node to the sink or Base Station (BS), some data packets may lost due to some reason such as failure of node, dead node. Sometimes node is not interested in forwarding data and drops the data packet. The problem of lost data affects the reliability of the network. Various energy efficient protocols have been proposed but they do not ensure reliability. This paper studies the reliability issue. Also highlights some of the proposed techniques to make network more reliable.
\end{abstract}

Keywords: WSN, SPIN, Data Centric, Reliability.

\section{Introduction}

Wireless sensor network (WSN) is widely use in different applications like environment, defense, medical, house, media and education. Wireless sensor network is a network of large amount of sensor nodes which are densely deployed in an ad-hoc manner. Figure 1 shows the structure of wireless sensor network in which source node transfers data to the Base Station (BS) with the help of some intermediate nodes and user access that data with the help of internet. It plays an important role in business and also provides alerts at the time of environmental disaster. These [1] sensors are capable of sensing particular condition and are configured for certain action. These sensors are able to communicate over limited range through radio waves. Wireless sensor nodes have four basic units named as sensing unit, processing unit, transmission unit and power unit. Figure 2 shows the detailed structure of sensor node. Sensing unit has two subunits known as sensor and Analog to Digital Converter (ADC). Sensor senses the situation and makes some analog signal which converts to digital signal by using $\mathrm{ADC}$ and then transfers it to the processing unit. According to that signal, a particular action is processed by the processing unit. If there is any need of data transmission to another node then it is done by the transmission unit. The whole node is powered by the power unit. Sensor network does not have any central point of control.

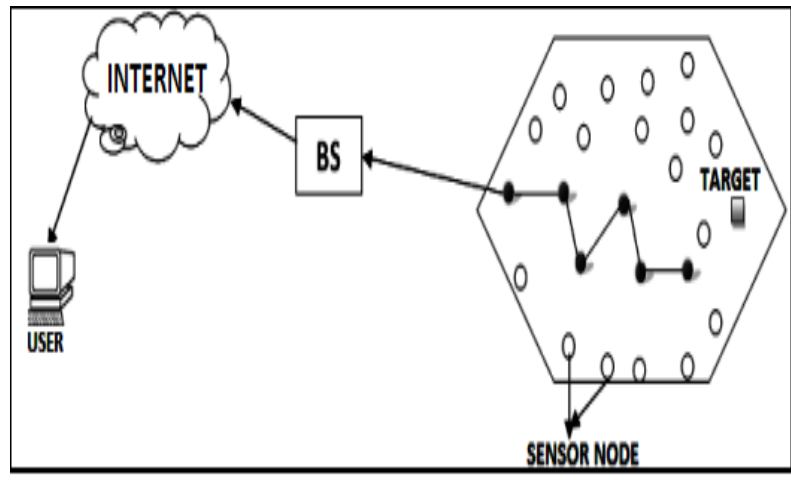

Fig 1: Wireless Sensor Network 


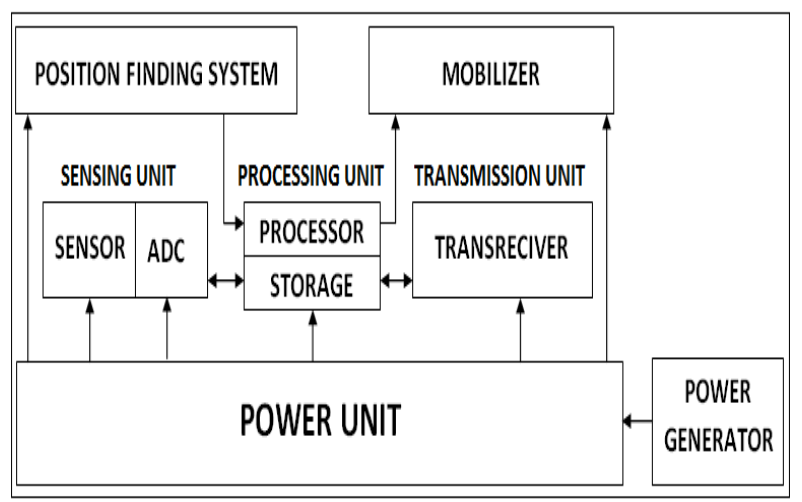

Fig 2: Detail Structure of Single Sensor Node

For transmitting data form source node to the base station various routing protocols have been proposed. These routing protocols are categorized into three types such as data centric protocols, hierarchical protocols and location based protocols.

WSN has various challenges [2]. To make network reliable is one of the main challenge in WSN. Reliability in a network means data should deliver to the destination without any loss. In sensor networks data loss occurs because of different reasons such as node becomes dead due to energy depletion or may be node is not interested to send data further and drop the data packet [3]. Many applications need a reliable protocol to fulfill its requirement. For example using sensors in an emergency area like to sense the fire has to be reliable because delay or lost information may be dangerous.

This paper focuses on the issues of SPIN (Sensor Protocol for Information via Negotiation) [4] [5] routing protocol. Spin is a data centric routing protocol. It is an energy efficient protocol and removes the problems of implosion, overlap and resource blindness. Many versions of SPIN have been proposed by the different researchers to overcome the problems of sensor networks but reliability is still a big issue to be resolved. The rest of the paper is organized as follows: Section II presents some different versions of SPIN protocol. Section III gives an overview of improvement over SPIN. Finally Section IV concludes the paper.

\section{Spin Family}

SPIN is a data centric protocol. In data centric routing, sink sends queries to certain regions and waits for the data from the sensors located in the selected regions. In SPIN, sensor nodes communicate about the data they already have and they still need to obtain.

SPIN (Sensor Protocol for Information via Negotiation): SPIN is an adaptive protocol which spreads all its information to the neighbor nodes in the network. This protocol enables the user for querying and getting information immediately. This protocol works in three steps [6] as:

- ADV: The sensor nodes broadcasts an ADV packet about the data to all its neighbors i.e. Meta data as shown in Figure 3.

- REQ: If any neighbor is interested in the data, it sends a REQ packet to the originator of ADV packet for the DATA.

- DATA: Data is sent to its neighbor without considering the type of data or what kind of data is required. By repeating the same the interested sensor nodes in the entire sensor network gets a copy of the data.

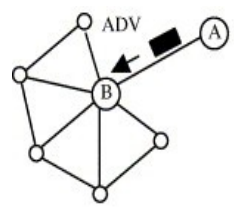

(a)

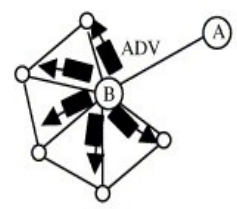

(d)

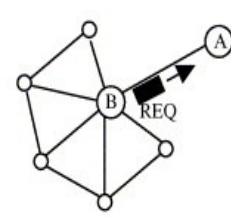

(b)

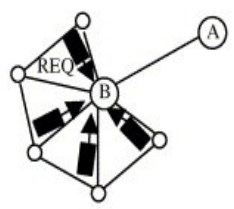

(e)

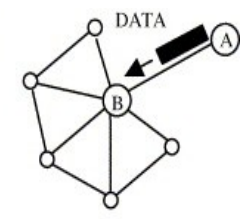

(c)

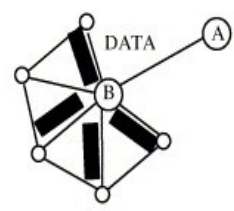

(f) 
(a) Node B responds by sending a request to Node A.

(b) After receiving the requested data

(c) Node B then sends out advertisements to its neighbors

(d) Who in turn send requests back to $\mathrm{B}(\mathrm{e}-\mathrm{f})$

Fig 3: Working of SPIN Protocol

Some proposed versions of SPIN routing protocols are described as follows:

SPIN-1: SPIN-1 overcomes the problem of blind forward and data inaccessible [7]. Its working is similar as that of SPIN but it has some better features than SPIN as mentioned below:

- The initial energy of each node is equal. Nodes A and B can communicate with each other, if link is symmetrical;

- Communication between two nodes is far away from the interference of other nodes, and power is without any constraints and nodes remain stable.

- Assuming all nodes want to achieve the data, and are located on the path to reach sink nodes.

- Wireless signals in all directions consume the same energy.

SPIN-1 compares the energy of the node when it REQ for data to threshold energy if energy is enough to perform whole process it sends 1 otherwise it will not respond to the ADV packet. But in SPIN, node sends 0 with REQ on ADV.

M-SPIN: Modified SPIN is energy efficient routing protocol where [8] node finds all information of its neighbors and find out the distance to the sink. Data is transfer to those nodes which are in the direction of sink rather than all neighbor nodes. Distance between nodes to the sink is calculated on the basis of hop distance. It performs in following 3-steps:

- Distance Discovery

- Negotiation

- Data Transmission

Optimized-Spin: Optimized SPIN [9] is more energy efficient than SPIN protocol. In Optimized SPIN, when any data is aggregated at the node, it broadcasts the ADV packet to its neighbor about the available data (Meta data) and node sends request REQ of the data according to its requirement. Advertised node sends only the requested data back to the node. Figure 4 shows the working of Optimized SPIN.

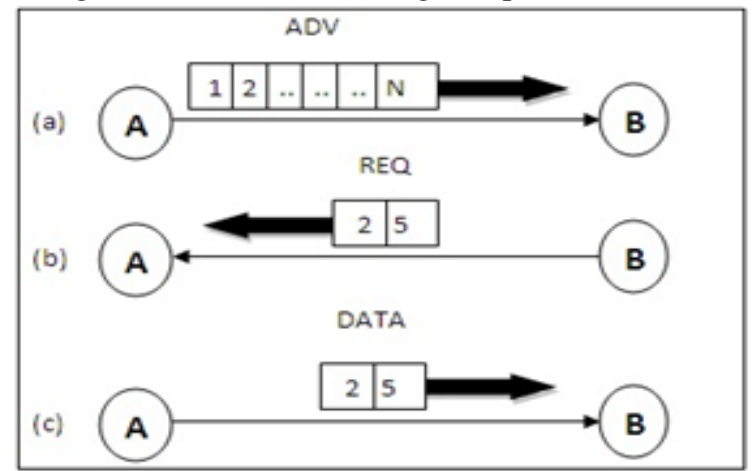

(a) Node A packet ADV having Meta-Data to B.

(b) Node B sends packet REQ having selected data request $2 \& 5$ to A.

(c) Node A sends only requested data $2 \& 5$ in Data Packet DATA to B.

Fig 4: Packet Transfer in Optimized SPIN Protocol

\section{Improvement Over Spin}

The comparison in data centric protocols in respect of parameters like average energy consumption, lifetime of nodes, average time taken to travel from source node to sink node, average number of nodes participated in the network are shown in [10]. The protocols which are compared are Flooding Mechanism, SPIN (Sensor Protocol for Information via Negotiation), MFCA (Minimum Cost Forwarding Algorithm). Out of these three, Flooding Mechanism achieves best performance because a node sends out the received data or the management packets to its neighbors by broadcasting, unless a maximum number of hops for that packet are reached or the destination of the packets is arrived but it has some problems like implosion, overlap and resource blindness. SPIN removes the problem of flooding but do not provide good performance. MFCA send data in to the direction of the sink where algorithm estimate the cost of data reached to sink. 
The concept of end to end acknowledgment at transport layer is suggested in [11]. When data is to be transmitted between source node to the destination, there should be an acknowledgement of data in between the middle of nodes. Thus, data will reach to the sink in reliable format without any loss. This mechanism performs with the help of MAC automatic repeat request. When a middle node transfers data to other intermediate node, they acknowledged the data by ACK/NACK. So, in this way, the loss of data will reduce and quality of data increased.

In [12], authors proposed idea of PEQ (periodic, event driven and query based) algorithm in direct diffusion data centric protocol and simulated on network simulator (NS-2). PEQ is a routing algorithm, which works in three steps. First step is constructing the hop tree, and then involves utilization of sensors and last step is responsible for data transmission.

- $\quad$ Subscribe Paradigm for Sensors

- $\quad$ Building the Hop Tree

- The Subscription Message Propagation

- Sending the Notification Message

- $\quad$ Path Repair Mechanism

Source floods the notification message and all possible path information to the sink. Sink select one path from this information and send reply from that path for receiving the data. If any node becomes dead then sink send reply to another path and source will send whole data again.

In [13], authors proposed a Modified SPIN for Wireless Sensor Networks. This paper shows that whenever data is to be transferred, it should be deliver to those nodes which are nearer to the sink and whose hop count is few which is calculated from distance discovery to save energy of nodes. The three phases of modified spin are:

- Distance Discovery

- Negotiation

- Data Transmission

This protocol is faster than the basic SPIN protocol as it avoids unnecessary flooding of data to those nodes which have higher hop count.

Corroborative Aggregation Protocol (CAP) is proposed in [14], in which, each sensor that detects a report from its neighbour that contradicts its own findings generates its own report to dispute the faulty report. The numbers of correct reports in the event are used to overcome adverse effects that may arise due to the wrong reports. CAP helps to improve the credibility of the fused content in the presence of faulty sensors. CAP works on the principle of having multiple sensors corroborate each other.

In this paper, author has proposed a new protocol SPROID (Scalable Protocol for Robust Information Dissemination) [15] [16] for efficient and reliable data delivery over the SPIN (Sensor Protocol for Information via Negotiation). SPROID achieve reliability by building a correct neighbour list in finite time. This protocol work as: suppose node A has its neighbour and node B. Node B have its neighbour and node A. When node A have data and broadcast the advertise packet about data. Node B request for data and got that data. Now node B advertises the message about data which received by the node $\mathrm{A}$ and match that information to its own stored data. If match successful then it will be acknowledgement otherwise node A sends data again to node B.

\section{Conclusion}

Wireless sensor network is widely using in today's time. Various routing protocols have been designed to overcome the challenges of the sensor network. The basic purpose of the routing protocol is to transmit data from source to sink but sometimes data got lost in between the path. This arise the issue of reliability. A data centric protocol known as SPIN protocol is explained in this paper with its different versions which fulfill the issue of energy, overlap, implosion and resource blindness. The improvements done by the researchers are highlighted to make a protocol more reliable.

\section{References}

[1] Martin Enzinger, "Energy-efficient communication in Wireless Sensor Networks", Proceedings of the Seminar Sensor Nodes Operation, Network and Application (SN), Summer Semester 2012, volume NET-2012-08-2 of Network Architectures and Services (NET), pp. 25-31, August 2012.

[2] Jyoti, Harkesh Sehrawat and Devender Sharma, "Energy Efficient M-SPIN Protocol”, International Journal of Scientific \& Engineering Research, Volume 3, Issue 10, pp. 1-9, October 2012.

[3] K. Vinoth Kumar and S.Karthikeyan, "Multihop Energy Efficient Reliable and Fault Tolerant Routing Protocol for Wireless Sensor Networks" International Journal of Emerging Technology and Advanced Engineering, Volume 3, Issue 2, February 2013.

[4] Nihal Srivastava, Sandeep Seth, Mohit Kumar and Rakesh Kumar, "Energy Efficient Protocols for Wireless Sensor Networks: A Review”, International Journal of Computer Technology \& Applications, Volume 4, Issue 3, pp. 434-444, May-June 2013.

[5] Jamal N. Al-Karaki and Ahmed E. Kamal, "Routing Techniques in Wireless Sensor Networks: A Survey" IEEE Conference on Wireless Communications, Volume 11, Issue 6, pp. 6-28, December 2004 
[6] Parul Tyagi and Surbhi Jain, "Comparative Study of Routing Protocols in Wireless Sensor Network" International Journal of Advanced Research in Computer Science and Software Engineering, Volume 2, Issue 9, pp. 134-140, September 2012.

[7] K. Karthikeyan and M.Kavitha, "Comparative Analysis of Data Centric Routing Protocols for Wireless Sensor Networks" International Journal of Scientific and Research Publications, Volume 3, Issue 1, pp. 1-6, January 2013.

[8] Zeenat Rehna, Sarbani Roy and Nandini Mukherjee, "A Modified SPIN for Wireless Sensor Network", Third International Conference on Communication Systems and Networks (COMSNETS), Volume 4, pp. 1-4, January 2011.

[9] Neha Khurana, Avadhesh Verma and Jitender Grover, "Optimized SPIN in Wireless Sensor Network", proceedings of IEEE International Conference on Human Computer Interactions (ICHCI'13), August 2013.

[10] T. Saravanan, G. Saritha and V. Srinivasan, "A Analysis of Flat Routing Protocols in Sensor Network", Middle-East Journal of Scientific Research, Volume 20, Issue 12, pp. 2566-2570, 2014.

[11] Paulo Rogerio Pereira, Antonio Grilo, Francisco Rocha, Mario Serafim Nunes, Augusto Casaca, Claude Chaudet, Peter Almstrom and Mikael Johansson, "End-to-End Reliability in Wireless Sensor Networks: Survey and Research Challenges", EuroFGI Workshop on IP QoS and Traffic Control, P. Pereira (Ed.), Lisbon, Portugal, pp. 67-74, December 2007.

[12] Azzedine Boukerche, Richard Werner Nelem Pazzi and Regina Borges Araujo, "A Fast and Reliable Protocol for Wireless Sensor Networks in Critical Conditions Monitoring Applications", 7th ACM international symposium on Modeling, analysis and simulation of wireless and mobile systems, pp 157-164, 2004.

[13] Wei Yuan, Srikanth V. Krishnamurthy, and Satish K. Tripathi, "Improving the Reliability of Event Reports in Wireless Sensor Networks", Ninth IEEE International Symposium on Computers and Communications, Volume 1, pp. 220-225, July 2004.

[14] Hari Rangarajan, J. J. and Garcia-Luna-Aceves, "Reliable Data Delivery in Event-Driven Wireless Sensor Networks", Ninth IEEE International Symposium on Computers and Communications, Volume 1, pp. 232-237, July 2004.

[15] Rajendra Singh Bisht and Shailendra Mishra, "Network Structure and Topology based Routing Techniques in Wireless Sensor Network - A survey", International Journal of Computer Applications, Volume 74, Issue 12, July 2013.

[16] Mohammed Omari, Noura Tiouririne, and Djamila Dahmani, "Simulation of the TEEN and the SPIN Protocols in a Wireless Sensor Network Environment", Cyber Journals: Multidisciplinary Journals in Science and Technology, Journal of Selected Areas in Telecommunications (JSAT), pp. 9-15, October 2012. 